\title{
The Variation Detracting Property of some Shannon Sampling Series and their Derivatives
}

\author{
Andi Kivinukk \\ Department of Mathematics, \\ Tallinn University, Narva Road 25 \\ Tallinn, 10120, Estonia \\ Email: andik@tlu.ee
}

\author{
Tarmo Metsmägi \\ Department of Mathematics, \\ Tallinn University, Narva Road 25 \\ Tallinn, 10120, Estonia \\ Email: tmetsmag@tlu.ee
}

\begin{abstract}
In this paper are considered some generalized Shannon sampling operators which preserve the total variation of functions and their derivatives. For that purpose will be used the averaged kernel functions of certain even bandlimited kernel functions.
\end{abstract}

\section{INTRODUCTION}

In this paper we investigate the generalized Shannon sampling operators, which preserve the total variation of functions and their derivatives.

Dealing with the class of functions of bounded variation $B V[0,1]$, the Bernstein polynomials have the following the total variation preserving property (due to G.G.Lorentz, 1937)

$$
V_{[0,1]}\left[B_{n} f\right] \leq V_{[0,1]}[f],
$$

where $f \in B V[0,1]$. In [3] this was called as the variation detracting property, sometimes called also as the variational diminishing property. Such kind of the total variation preserving property is known for many positive operators [1].

There have been also interests in the variation detracting property for the derivative of the Bernstein operator (e.g. [5]) expressed by the inequality

$$
V_{[0,1]}\left[\left(B_{n} f\right)^{\prime}\right] \leq V_{[0,1]}\left[f^{\prime}\right] .
$$

The generalized Shannon sampling operators [4] for the uniformly continuous and bounded functions $f \in C(\mathbb{R})$ are given by $(t \in \mathbb{R} ; W>0)$

$$
\left(S_{W} f\right)(t):=\sum_{k=-\infty}^{\infty} f\left(\frac{k}{W}\right) s(W t-k) .
$$

The variation detracting property for the generalized Shannon sampling operators could be in form:

let $f \in B V(\mathbb{R})$ implies $S_{W} f \in B V(\mathbb{R})$ and

$$
V_{\mathbb{R}}\left[S_{W} f\right] \leq C_{0}\left(S_{W}\right) V_{\mathbb{R}}[f]
$$

is valid, where the constant $C_{0}=C_{0}\left(S_{W}\right)$ depends on the norm of the operator $S_{W}: C(\mathbb{R}) \rightarrow C(\mathbb{R})$. The variation detracting property for the derivatives of the generalized Shannon sampling operators could be in form:

let $f^{\prime} \in B V(\mathbb{R})$ implies $\left(S_{W} f\right)^{\prime} \in B V(\mathbb{R})$ and

$$
V_{\mathbb{R}}\left[\left(S_{W} f\right)^{\prime}\right] \leq C_{1}\left(S_{W}\right) V_{\mathbb{R}}\left[f^{\prime}\right]
$$

for some constant $C_{1}=C_{1}\left(S_{W}\right)$ depending on the norm of the operator $S_{W}$.

For any $f \in C(\mathbb{R})$ the operators $S_{W}$ are well-defined, if the kernel function $s$ satisfies the condition

$$
\sup _{u \in \mathbb{R}} \sum_{k=-\infty}^{\infty}|s(u-k)|<\infty
$$

hence $s \in L^{1}(\mathbb{R})$. Moreover [4], $\left\{S_{W}\right\}_{W>0}$ defines a family of bounded linear operators from $C(\mathbb{R})$ into itself, having its operator norm

$$
\left\|S_{W}\right\|=\sup _{u \in \mathbb{R}} \sum_{k=-\infty}^{\infty}|s(u-k)| \quad(W>0) .
$$

In our approach the kernel functions of sampling operators defined above will be some even band-limited functions $s$, i.e. $s \in L^{1}(\mathbb{R})$, and these are given as the Fourier transform of an even window function $\lambda \in C_{[-1,1]}, \lambda(0)=1, \lambda(u)=0$ $(|u| \geqslant 1)$ by the equality

$$
s(t):=s(\lambda ; t):=\int_{0}^{1} \lambda(u) \cos (\pi t u) d u .
$$

These type of window (also called as the apodization) functions have been widely used in applications (e.g., [2], [10] and literature cited there), in Signal Analysis in particular, very long time.

The leading idea to consider the variation detracting property of (1) is to construct some related kernels to the kernel (4). For the kernels defined by (4) holds the following proposition.

Proposition 1. Define the related kernels to the kernel (4) as follows:

$$
s_{m}(t):=\int_{0}^{1} \frac{\lambda(u)}{\operatorname{sinc}(m u)} \cos (\pi t u) d u
$$

for $0<m \leq 1$, and

$$
s_{m, n}(t):=\int_{0}^{1} \frac{\lambda(u)}{\operatorname{sinc}(m u) \operatorname{sinc}(n u)} \cos (\pi t u) d u
$$


for $0<m, n \leq 1$. Then

$$
\begin{aligned}
s(t) & =\frac{1}{2 m} \int_{-m}^{m} s_{m}(t+x) d x \\
& =\frac{1}{4 m n} \int_{-m}^{m} d x \int_{-n}^{n} s_{m, n}(t+x+y) d y \\
s_{m}(t) & =\frac{1}{2 n} \int_{-n}^{n} s_{m, n}(t+y) d y .
\end{aligned}
$$

Remark 1. The kernels (5) and (6) are well-defined, if in case $m=1$ or $n=1$ we assume the existence of the continuous left derivative with value $\lambda^{\prime}(1-)=0$. If $m=n=1$, we assume the existence of the continuous left second derivative with value $\lambda^{\prime \prime}(1-)=0$.

Since (8) and (9), we have the following

Corollary 1. If $s_{m, n} \in L^{1}(\mathbb{R})$, then $s_{m} \in L^{1}(\mathbb{R})$ and

$$
\|s\|_{1} \leq\left\|s_{m}\right\|_{1} \leq\left\|s_{m, n}\right\|_{1} \text {. }
$$

Remark 2. If $s \in L^{1}(\mathbb{R})$, then by (4) $s \in B_{\pi}^{1} \subset L^{1}(\mathbb{R})$, where $B_{\pi}^{1}$ is a Bernstein class [6]. Under conditions of Remark 1, and by Proposition 1, equations (5), (6), assuming $s_{m, n} \in L^{1}(\mathbb{R})$, the kernels $s_{m}, s_{m, n} \in B_{\pi}^{1}$ as well.

Some examples of kernels defined by (4), which appear in our applications, are given as follows:

1) $\lambda(u)=1$ defines the sinc function (the exceptional case, because $\left.\operatorname{sinc}(\cdot) \notin L^{1}(\mathbb{R})\right)$

$$
\operatorname{sinc}(t):=\frac{\sin \pi t}{\pi t}
$$

2) $\lambda(u)=\operatorname{sinc} u$ defines the Lanczos' kernel (with corresponding operator $L_{W}$ ), which by (4) appears to be the averaged sinc-function

$$
s_{L}(t)=\frac{1}{2} \int_{-1}^{1} \operatorname{sinc}(t+v) d v
$$

3) $\lambda(u)=\cos \frac{\pi u}{2}$ defines the Rogosinski-type kernel (with corresponding operator $R_{W}$ ) in the form

$$
r_{0}(t)=\frac{1}{2 \pi} \cdot \frac{\cos \pi t}{\frac{1}{4}-t^{2}}
$$

\section{TOTAL VARIATION ON $\mathbb{R}$}

Let us consider functions of bounded variation in the following meaning.

Definition. We say that $f \in B V(\mathbb{R})$, the space of all functions of bounded variation on $\mathbb{R}$, iff there exists a.e. derivative $f^{\prime} \in L^{1}(\mathbb{R})$; and we define the total variation of $f \in B V(\mathbb{R})$ as

$$
V_{\mathbb{R}}[f]:=\left\|f^{\prime}\right\|_{1}=\int_{-\infty}^{\infty}\left|f^{\prime}(v)\right| d v
$$

Next we give some statements and properties of $B V(\mathbb{R})$.

Proposition 2. For $f \in B V(\mathbb{R})$ and any monotone increasing sequence $\left\{t_{k}\right\}_{k=-\infty}^{\infty} \subset \mathbb{R}$ with $\lim _{k \rightarrow \pm \infty} t_{k}= \pm \infty$ one has:

1)

$$
\sum_{k=-\infty}^{\infty}\left|f\left(t_{k}+v\right)-f\left(t_{k-1}+v\right)\right| \leqslant V_{\mathbb{R}}[f] \quad(v \in \mathbb{R})
$$

2) $f$ is bounded and there exist $\lim _{t \rightarrow \pm \infty} f(t)$;

3) $f$ is locally integrable, and

$$
\frac{1}{b-a} \sum_{k=-\infty}^{\infty} \int_{a}^{b}\left|f\left(t_{k}+v\right)-f\left(t_{k-1}+v\right)\right| d v \leqslant V_{\mathbb{R}}[f] .
$$

Proposition 3. Let $f^{\prime} \in B V(\mathbb{R})$, and let $a \in \mathbb{R}, W>$ $0, t_{k}=\frac{k}{W}, k \in \mathbb{Z}$. Then

$W \sum_{k=-\infty}^{\infty}\left|f\left(t_{k}+a\right)-2 f\left(t_{k-1}+a\right)+f\left(t_{k-2}+a\right)\right| \leq V_{\mathbb{R}}\left[f^{\prime}\right]$.

\section{THE VARIATION DETRACTING PROPERTY}

The variation detracting property of certain sampling operators will be considered for $B V(\mathbb{R})$, the space of all functions of bounded variation on $\mathbb{R}$. This property is important in practice, since often signals are discontinuous but still with bounded variation.

Theorem 1. Assume $s_{m} \in L^{1}(\mathbb{R})$. If there exists a number $b \in \mathbb{R}$ such that $\pm m-b \in \mathbb{Z}$, then for $f \in B V(\mathbb{R})$ we have $S_{W} f \in B V(\mathbb{R})$ and

$$
V_{\mathbb{R}}\left[S_{W} f\right] \leq\left\|s_{m}\right\|_{1} V_{\mathbb{R}}[f] .
$$

The proof of Theorem 1 was essentially presented in [7]. By Corollary 1 and Theorem 1 we obtain under assumptions of Theorem 1

\section{Corollary 2.}

$$
V_{\mathbb{R}}\left[S_{W} f\right] \leq\left\|s_{m, n}\right\|_{1} V_{\mathbb{R}}[f] .
$$

For the proof of Theorem 2 we need the following technical lemma.

Lemma. For any sequence $\left(a_{0}, a_{1}, a_{2}, \ldots\right)$ and $M, N \in \mathbb{N}$ we have

$\sum_{i=1}^{M} \sum_{j=1}^{N}\left(a_{i+j}-2 a_{i+j-1}+a_{i+j-2}\right)=a_{0}-a_{M}-a_{N}+a_{M+N}$.

The variation detracting property for derivatives will be read as follows.

Theorem 2. Assume the kernel $s_{m, n} \in L^{1}(\mathbb{R})$ for $m \neq$ $0, n \neq 0$, such that there exists $b \in \mathbb{R}$ with $\pm m \pm n-b \in \mathbb{Z}$. If $f$ is bounded and $f^{\prime} \in B V(\mathbb{R})$, then $\left(S_{W} f\right)^{\prime} \in B V(\mathbb{R})$ and

$$
V_{\mathbb{R}}\left[\left(S_{W} f\right)^{\prime}\right] \leq\left\|s_{m, n}\right\|_{1} V_{\mathbb{R}}\left[f^{\prime}\right]
$$

Proof. By (7) we get (see also Remarks 1 and 2)

$$
s^{\prime}(t)=\frac{1}{2 m}\left(s_{m}(t+m)-s_{m}(t-m)\right)
$$


and hence by (9) we obtain

$$
\begin{aligned}
& s^{\prime \prime}(t)=\frac{1}{2 m}\left(s_{m}^{\prime}(t+m)-s_{m}^{\prime}(t-m)\right) \\
= & \frac{1}{4 m n} \int_{-n}^{n}\left(s_{m, n}^{\prime}(t+m+y)-s_{m, n}^{\prime}(t-m+y)\right) d y \\
= & \frac{1}{4 m n}\left(s_{m, n}(t+m+n)-s_{m, n}(t+m-n)\right. \\
- & \left.s_{m, n}(t-m+n)+s_{m, n}(t-m-n)\right) .
\end{aligned}
$$

So by (1) we have

$$
\begin{aligned}
\left(S_{W} f\right)^{\prime \prime}(t) & =W^{2} \sum_{k} f\left(\frac{k}{W}\right) s^{\prime \prime}(W t-k) \\
& =\frac{W^{2}}{4 m n} \sum_{k} f\left(\frac{k}{W}\right)\left(s_{m, n}(W t-k+m+n)\right. \\
& -s_{m, n}(W t-k+m-n) \\
& -s_{m, n}(W t-k-m+n) \\
& \left.+s_{m, n}(W t-k-m-n)\right) .
\end{aligned}
$$

Since $s_{m, n} \in L^{1}(\mathbb{R})$, then by (6) $s_{m, n} \in B_{\pi}^{1} \subset L^{1}(\mathbb{R})$ and for $s_{m, n}$ the condition (2) is satisfied by Nikolskii's inequality. Therefore, since $f$ is bounded, the series here is absolutely and uniformly convergent. Under assumptions of Theorem 2 on a number $b \in \mathbb{R}$ we get

$$
\begin{aligned}
& \left(S_{W} f\right)^{\prime \prime}(t) \\
= & \frac{W^{2}}{4 m n} \sum_{k}\left(f\left(\frac{k+m+n-b}{W}\right)-f\left(\frac{k+m-n-b}{W}\right)\right. \\
- & \left.f\left(\frac{k-m+n-b}{W}\right)+f\left(\frac{k-m-n-b}{W}\right)\right) \\
\times \quad & s_{m, n}(W t-k+b) .
\end{aligned}
$$

The application of Lemma with $M=2 m, N=2 n$,

$$
a_{l}=f\left(\frac{k+l-m-n-b}{W}\right),
$$

$(k \in \mathbb{Z}, W>0, l=0,1, \ldots)$ gives

$$
\begin{aligned}
& f\left(\frac{k-m-n-b}{W}\right)-f\left(\frac{k+m-n-b}{W}\right) \\
-\quad & f\left(\frac{k-m+n-b}{W}\right)+f\left(\frac{k+m+n-b}{W}\right) \\
= & \sum_{i=1}^{2 m} \sum_{j=1}^{2 n}\left(f\left(\frac{k+i+j-m-n-b}{W}\right)\right. \\
- & 2 f\left(\frac{k+i+j-1-m-n-b}{W}\right) \\
+ & \left.f\left(\frac{k+i+j-2-m-n-b}{W}\right)\right) .
\end{aligned}
$$

Now by (11) we have

$$
\begin{gathered}
\left(S_{W} f\right)^{\prime \prime}(t)=\frac{W^{2}}{4 m n} \sum_{k} \sum_{i=1}^{2 m} \sum_{j=1}^{2 n}\left(f\left(\frac{k+i+j-m-n-b}{W}\right)\right. \\
-2 f\left(\frac{k+i+j-1-m-n-b}{W}\right) \\
\left.+f\left(\frac{k+i+j-2-m-n-b}{W}\right)\right) s_{m, n}(W t-k+b) .
\end{gathered}
$$

Estimating and integrating over $\mathbb{R}$ yields

$$
\begin{array}{r}
\left\|\left(S_{W} f\right)^{\prime \prime}\right\|_{1} \leq\left\|s_{m, n}\right\|_{1} \frac{W}{4 m n} \sum_{i=1}^{2 m} \sum_{j=1}^{2 n} \sum_{k} \mid f\left(\frac{k}{W}+a_{i j}\right) \\
-2 f\left(\frac{k-1}{W}+a_{i j}\right)+f\left(\frac{k-2}{W}+a_{i j}\right) \mid,
\end{array}
$$

where $a_{i j}=\frac{i+j-m-n-b}{W}$. The application of Proposition 3 yields

$$
\begin{aligned}
\left\|\left(S_{W} f\right)^{\prime \prime}\right\|_{1} & \leq\left\|s_{m, n}\right\|_{1} \frac{1}{4 m n} \sum_{i=1}^{2 m} \sum_{j=1}^{2 n} V_{\mathbb{R}}\left[f^{\prime}\right] \\
& =\left\|s_{m, n}\right\| V_{\mathbb{R}}\left[f^{\prime}\right] .
\end{aligned}
$$

This concludes the proof.

In analogous way we can consider the variation detracting property for the second derivative. We have the following

Theorem 3. Assume the kernel $s_{m, n, r} \in L^{1}(\mathbb{R})$ for

$0<m, n, r<1$ such that there exists $b \in \mathbb{R}$ with $\pm m \pm n \pm r-b \in \mathbb{Z}$. Here

$$
s_{m, n, r}(t):=\int_{0}^{1} \frac{\lambda(u)}{\operatorname{sinc}(m u) \operatorname{sinc}(n u) \operatorname{sinc}(r u)} \cos (\pi t u) d u \text {. }
$$

If $f$ is bounded and $f^{\prime \prime} \in B V(\mathbb{R})$, then $\left(S_{W} f\right)^{\prime \prime} \in B V(\mathbb{R})$ and

$$
V_{\mathbb{R}}\left[\left(S_{W} f\right)^{\prime \prime}\right] \leq\left\|s_{m, n, r}\right\|_{1} V_{\mathbb{R}}\left[f^{\prime \prime}\right]
$$

\section{Applications}

1) If we take $\lambda_{L}(u)=\operatorname{sinc} u$, the Lanczos' window function, then we get

$$
\frac{\lambda_{L}(u)}{\operatorname{sinc} \frac{u}{2}}=\cos \frac{\pi u}{2} \equiv \lambda_{R}(u),
$$

which is the Rogosinski window. Applying Theorem 1 with $m=b=\frac{1}{2}$, by Corollary 2 we obtain

$$
V_{\mathbb{R}}\left[L_{W} f\right] \leq\left\|r_{0}\right\|_{1} V_{\mathbb{R}}[f] .
$$

If we take $m=n=\frac{1}{2}$ in (6), we get

$$
\frac{\lambda_{L}(u)}{\operatorname{sinc}^{2} \frac{u}{2}}=\frac{\pi u}{2} \cot \frac{\pi u}{2} \equiv \lambda_{F_{1}}(u)
$$

which defines the Favard-type kernel $s_{F_{1}}$ ([9], Section 4.1.3; [11], Chapter 3, Section 1). Applying Theorem 2 with $m=n=\frac{1}{2}, b=0$, we obtain

$$
V_{\mathbb{R}}\left[\left(L_{W} f\right)^{\prime}\right] \leq\left\|s_{F_{1}}\right\|_{1} V_{\mathbb{R}}\left[f^{\prime}\right]
$$


If we take $m=n=r=\frac{1}{2}$ in Th. 3, then we get

$$
\frac{\lambda_{L}(u)}{\operatorname{sinc}^{3} \frac{u}{2}}=\left(\frac{\pi u}{2}\right)^{2} \frac{\cot \frac{\pi u}{2}}{\sin \frac{\pi u}{2}} \equiv \lambda_{F_{2}}(u),
$$

which defines the Favard-type kernel $s_{F_{2}}$ (see [11], Ch. 3, Sect. 1). Applying Theorem 3 with $m=n=r=b=\frac{1}{2}$, we obtain

$$
V_{\mathbb{R}}\left[\left(L_{W} f\right)^{\prime \prime}\right] \leq\left\|s_{F_{2}}\right\|_{1} V_{\mathbb{R}}\left[f^{\prime \prime}\right] .
$$

Remark 3. In [8] we proved that $\left\|r_{0}\right\|_{1}=\left\|L_{W}\right\|$, thus the variation detracting property takes a very natural shape,

$$
V_{\mathbb{R}}\left[L_{W} f\right] \leq\left\|L_{W}\right\| V_{\mathbb{R}}[f] .
$$

2) If we take $\lambda_{R}(u)=\cos \frac{\pi u}{2}$, the Rogosinski window function, we get

$$
\frac{\lambda_{R}(u)}{\operatorname{sinc} \frac{u}{2}}=\lambda_{F_{1}}(u)
$$

Applying Theorem 1 with $m=b=\frac{1}{2}$, we get

$$
V_{\mathbb{R}}\left[R_{W}\right] \leq\left\|s_{F_{1}}\right\|_{1} V_{\mathbb{R}}[f] .
$$

If we take $m=n=\frac{1}{2}$ in (6), we again have the Favard-type window

$$
\frac{\lambda_{R}(u)}{\operatorname{sinc}^{2} \frac{u}{2}}=\left(\frac{\pi u}{2}\right)^{2} \cdot \frac{\cot \frac{\pi u}{2}}{\sin \frac{\pi u}{2}}=\lambda_{F_{2}}(u) .
$$

Applying Theorem 2 with $m=n=\frac{1}{2}, b=0$, we have

$$
V_{\mathbb{R}}\left[\left(R_{W} f\right)^{\prime}\right] \leq\left\|s_{F_{2}}\right\|_{1} V_{\mathbb{R}}\left[f^{\prime}\right]
$$

3) Let $\lambda_{H}(u)=\cos ^{2} \frac{\pi u}{2}$ be the Hann window function, then

$$
\frac{\lambda_{H}(u)}{\operatorname{sinc} u}=\frac{\pi u}{2} \cot \frac{\pi u}{2}=\lambda_{F_{1}}(u) .
$$

Applying Theorem 1 with $m=b=1$ we get

$$
V_{\mathbb{R}}\left[H_{W} f\right] \leq\left\|s_{F_{1}}\right\|_{1} V_{\mathbb{R}}[f] .
$$

If we take $m=1, n=\frac{1}{2}$ in (6), we have

$$
\frac{\lambda_{H}(u)}{\operatorname{sinc} u \cdot \operatorname{sinc} \frac{u}{2}}=\frac{\cos \frac{\pi u}{2}}{\operatorname{sinc}^{2} \frac{u}{2}}=\lambda_{F_{2}}(u),
$$

which defines the Favard-type kernel. Applying Theorem 2 with $m=1, n=\frac{1}{2}, b=\frac{1}{2}$, we obtain

$$
\begin{gathered}
V_{\mathbb{R}}\left[\left(H_{W} f\right)^{\prime}\right] \leq\left\|s_{F_{2}}\right\|_{1} V_{\mathbb{R}}\left[f^{\prime}\right] . \\
\text { V. CONCLUSION }
\end{gathered}
$$

We investigated the variation detracting property of the generalized Shannon sampling operators,

$$
\left(S_{W} f\right)(t):=\sum_{k=-\infty}^{\infty} f\left(\frac{k}{W}\right) s(W t-k),
$$

which preserve the total variation of functions and their derivatives, i.e.

$$
V_{\mathbb{R}}\left[\left(S_{W} f\right)^{(k)}\right] \leq\left\|s_{m, n, r}\right\|_{1} V_{\mathbb{R}}\left[f^{(k)}\right],
$$

where $k=0,1,2$ and $s_{m, n, r}$ are certain related kernels to the original kernel

$$
s(t):=s(\lambda ; t):=\int_{0}^{1} \lambda(u) \cos (\pi t u) .
$$

As applications we considered some, in literature known examples of kernels, which realize the variation detracting property of the generalized Shannon sampling operators.

\section{ACKNOWLEDGMENT}

This research was partially supported by the Estonian Sci. Foundation, grant 8627 and by Estonian Center of Excellence "Mesosystems - Theory and Applications", AU/8211.

The authors are grateful to their colleague G. Tamberg (Tallinn University of Technology) for some ideas concerning the kernels $s_{m}$.

\section{REFERENCES}

[1] J. A. Adell and J. de la Cal, Bernstein-type operators diminish the $\varphi$ variation, Constr. Approx., 12: 489-507, 1996.

[2] H. H. Albrecht, A family of cosine-sum windows for high resolution measurments. In IEEE International Conference on Acoustics, Speech and Signal Processing, Salt Lake City, May 2001, pages 3081-3084. Salt Lake City, 2001.

[3] C. Bardaro, P. L. Butzer, R. L. Stens and G. Vinti, Convergence in variation and rates of approximation for Bernstein-type polynomials and singular convolution integrals, Analysis ( Munich), 23: 299-346, 2003.

[4] P. L. Butzer, W. Splettstösser and R. L. Stens, The sampling theorems and linear prediction in signal analysis. Jahresber.Deutsch. Math-Verein 90:1-70, 1988

[5] T. N. T. Goodman, Variation diminishing properties of Bernstein polynomials on triangles, J. Approx. Theory, 50: 111-126, 1987.

[6] J. R. Higgins, Sampling Theory in Fourier and Signal Analysis, Clarendon Press, Oxford, 1996.

[7] A. Kivinukk, On some Shannon sampling series with the variation detracting property, Proc. of the 9th Intern. Conf. on Sampling Theory and Applications, Singapore, May 2-6, 2011, A. Khong, F. Oggier (Eds.), Nanyang Technological University, Singapore 2011, 1 - 4

[8] A. Kivinukk and G. Tamberg, On sampling series based on some combinations of sinc fubctions, Proc. of the Estonian Academy of Sciences. Physics Mathematics, 51: 203-220, 2002.

[9] N. P. Korneichuk, Exact Constants in Approximation Theory. Cambridge Univ. Press, 1991.

[10] H. D. Meikle, A New Twist to Fourier Transforms. Dover, Berlin, 2004.

[11] V. V. Zhuk, Approximation of Periodic Functions. (Russ.) Leningrad Univ. Press, Leningrad, 1982. 